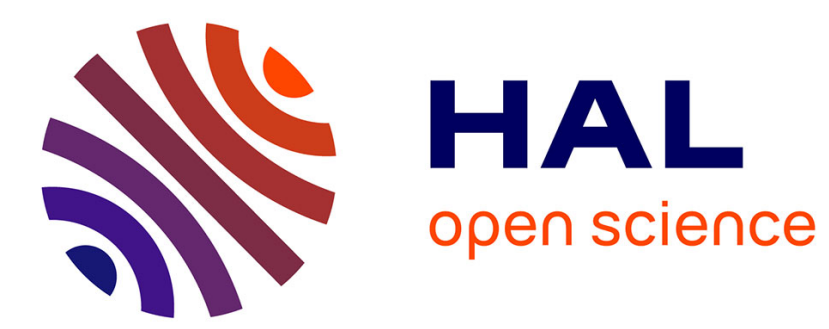

\title{
DIFFUSION ELASTIQUE, INELASTIQUE ET QUASI ELASTIQUE
}

\author{
Nicolas C.M. Marty
}

\section{To cite this version:}

Nicolas C.M. Marty. DIFFUSION ELASTIQUE, INELASTIQUE ET QUASI ELASTIQUE. Journal de Physique Colloques, 1970, 31 (C2), pp.C2-58-C2-64. 10.1051/jphyscol:1970207 . jpa-00213764

\section{HAL Id: jpa-00213764 https://hal.science/jpa-00213764}

Submitted on 1 Jan 1970

HAL is a multi-disciplinary open access archive for the deposit and dissemination of scientific research documents, whether they are published or not. The documents may come from teaching and research institutions in France or abroad, or from public or private research centers.
L'archive ouverte pluridisciplinaire HAL, est destinée au dépôt et à la diffusion de documents scientifiques de niveau recherche, publiés ou non, émanant des établissements d'enseignement et de recherche français ou étrangers, des laboratoires publics ou privés. 


\section{DIFFUSION ELASTIQUE, INELASTIQUE ET QUASI ELASTIQUE}

N. Marty

Institut de Physique Nucléaire, B.P. $n^{\circ} 1,91$ - Orsay.

La question à laquelle je voudrais essayer de répondre est la suivante : les mécanismes de diffusion inélastique et quasi élastique sont-ils actuellement suffisamment bien décrits pour que, à partir de ces réactions, on puisse obtenir des renseignements sur les fonctions d'ondes microscopiques des noyaux. Nous ne considérerons que les expériences faites à une énergie telle que 1 'on soit sûr d'avoir affaire à un processus d'interaction directe.

I. Diffusion inélastique.- Pour traiter la diffusion inélastique, les deux êlêments principaux sont le choix de l'interaction à deux corps et la description de la distorsion. Aux énergies relativement basses ( 20 à $50 \mathrm{MeV}$ ), les effets de la distorsion sont très importants. Ils sont traités de façon assez complète en D.W.B.A. ou par un calcul en équations couplées ; par contre, la force à deux corps est représentée par une interaction relativement simple qui permet de mener à bien les ca1culs de distorsion. A énergies plus élevées (supérieures à $100 \mathrm{MeV}$ en protons), les effets de distorsion, moins importants, sont traités de façon plus approchée, mais on utilise,pour l'interaction à deux corps, la matrice de diffusion nucléonnucléon libre ; il reste les effets hors couche d'ênergie, mais pour des particules incidentes de plus de $100 \mathrm{MeV}$ et de faibles transferts d'impulsion au noyau, on peut les considérer comme négligeables.

Pour les calculs de distorsion, il faut évidemment bien connaître le potentiel optique reprêsentant le noyau. On peut considérer que,pour la diffusion de protons, on dispose d'un potentiel optique moyen dont les paramètres varient régulièrement avec 1 'énergie et qui rend bien compte des résu1tats expérimentaux pour les sections efficaces différentielles et les polarisations entre 30 et 180 $\mathrm{MeV}$ [1]. Les principales caractêristiques sont : une importance croissant avec l'ênergie de l'absorption en volume, un rayon du potentiel central imaginaire $R_{i}$ supérieur à celui du potentiel centra1 réel $R_{r}$, un rayon du potentiel spin orbite
$\mathrm{R}_{\mathrm{S} 0}$ nettement inférieur à $\mathrm{R}_{\mathrm{r}}$ et la nécessité pour les énergies élevées d'une partie imaginaire pour le potentiel spin orbite; il reste à déterminer de façon plus précise la dépendance de ce potentiel avec le spin isotopique; il semble que la forme du potentiel optique dépende davantage du nombre de neutrons que du nombre de protons; c'est ce qu'indiquent les mesures de sections efficaces totales de réaction faites par Menet et al. (communication); ceci peut s'expliquer par le fait que la force $p . n$ est plus importante que $1 a$ force p.p ; ce résultat peut aussi s'interprêter par une distribution de neutrons plus étendue que la distribution des protons.

Dans le cas où le niveau fondamental est fortement couplê à un niveau proche (premier niveau $2^{+}$ des noyaux déformés), on doit pouvoir obtenir une meilleure détermination du potentiel optique par un traitement en équations couplées du fondamental et du premier niveau excitê et peut-être diminuer les variations des paramètres du potentiel optique d'un noyau à un noyau voisin ; il n'y a pas,à ma connaissance, d'êtude d'ensemble sur ce point; mais un travail déjà ancien [2] sur $1 e^{28} \mathrm{Si}$ à $30 \mathrm{MeV}$ montre les différences entre potentiel optique déterminé uniquement à partir du fondamental et en équations couplées.

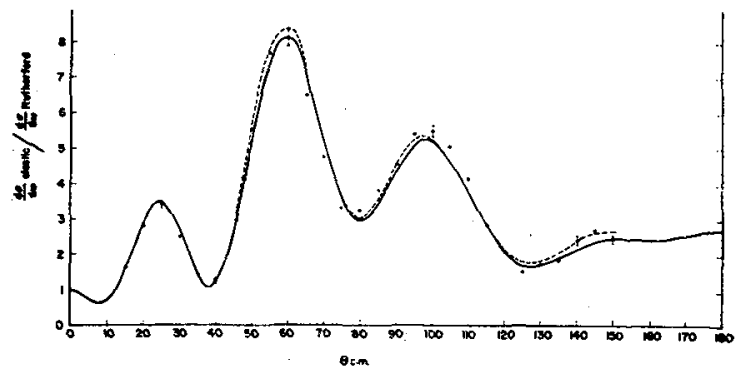

Rapport $\frac{\mathrm{d} \sigma / \mathrm{d} \Omega_{\text {êlas. }} \text { Fig. } 1}{\mathrm{d \sigma} / \mathrm{d} \Omega_{\text {Rutherford }}} ;$ - potentiel optique déterminé en équations couplées :--- potentiel optique habituel (Cole et al. [2]).

On ne fait jamais intervenir de termes d'interaction entre spin $\vec{\sigma}_{p}$ de la particule incidente et spin $\vec{\sigma}_{N}$ du noyau cible. Beurtey, catillon et 
Schnabel (comunication) se sont demandês si cela était justifié ; des différences pour les polarisations obtenues en diffusion élastique sur des noyaux voisins de spin très différent pouvaient être une indication de leur existence, mais les potentiels optiques, surtout pour les noyaux légers, varient d'un noyau à l'autre sans que cela soit encore bien expliqué. Les termes d'interaction $\vec{\sigma}_{\mathrm{P}} \vec{\sigma}_{\mathrm{N}}$ petits en tous cas devant les autres, sont totalement masqués dans des mesures de section efficace ; c'est dans une mesure du paramètre de dépolarisation $D$, au minimum de la section efficace de diffusion élastique faite sur ${ }^{27} \mathrm{Al},{ }_{\mathrm{B}}$ et ${ }^{9}$ Be que Beurtey et al. ont pu montrer leur existence mais aussi leur faible valeur.

Je ne discuterai pas ici de la D.W.B.A. ; les calculs faits à moyenne énergie avec cette approximation donnent des distributions angulaires en diffusion inélastique en accord avec les formes expérimentales, mais les valeurs absolues des sections efficaces sont toujours beaucoup trop faibles. Amos et al. [3] puis Schaeffer [4] ont amélioré les résultats par un traitement antisymétrisé de 1'amplitude de diffusion nucléon noyau qui a pour effet de multiplier la section efficace par un facteur important (qui peut atteindre 3 ou 4 [4]) sans en modifier la forme. Cependant le facteur multiplicatif dû aux termes d'êchange est extrêmement sensible au choix de la force utilisée pour représenter 1 'interaction à deux corps.

Nous touchons là au problème principal : le choix de la force à deux corps à utiliser ; il y a deux doctrines : I'une d'elles préconise l'utilisation d'ure force voisine ou'identique à celle qui a servi à 1 'établissement du modèle microscopique du noyau, 1'autre est pour 1 'utilisation de la force nucléon nucléon libre ; un argument donné en faveur de la seconde est que l'interaction a lieu essentiellement en surface et que le principe de Pauli intervient peu. Les deux doctrines se rapprochent si $I^{\prime}$ on arrive à une description des modèles microscopiques qui utilise des forces très proches des rorces nuclêon nuclến libre (potentiel de Tabakin ou d'Hamada Johnston).

Si l'on veut aller plus loin et expliquer non seulement les sections efficaces de diffusion inélastique mais ausi les polarisations, il faut, dans la force, tenir compte d'un terme d'interaction spin-orbite ou $d$ 'un potentiel $\mathrm{V}_{S O}$. Dans un calcul macroscopique en D.W.B.A. où les états excités sont obtenus dans un développement en fonction d'un paramètre de déformation du fondamental, Sherif et Blair [5] ont montrê que contrairement aux essais faits prêcêdemment, on rendait très bien compte des asymétries et des polarisations en diffusion $p, p^{\prime}$ en prenant comme potentiel spin-orbite la forme de Thomas complète

$$
v_{S 0}=\left(\frac{\hbar}{m_{\pi} c}\right)^{2} \vec{\sigma}\left(\vec{\nabla} \vee(\vec{r}) \wedge \frac{\vec{\nabla}}{i}\right)
$$

mais la partie spin-orbite doit avoir une déformation plus grande que la partie centrale $\left(\beta_{S 0}=1,5 \beta_{r}\right.$ ou $\left.\beta_{S O} R_{S O}=\beta_{r} R_{r}\right)$.

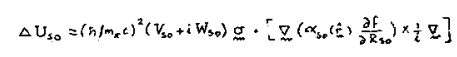

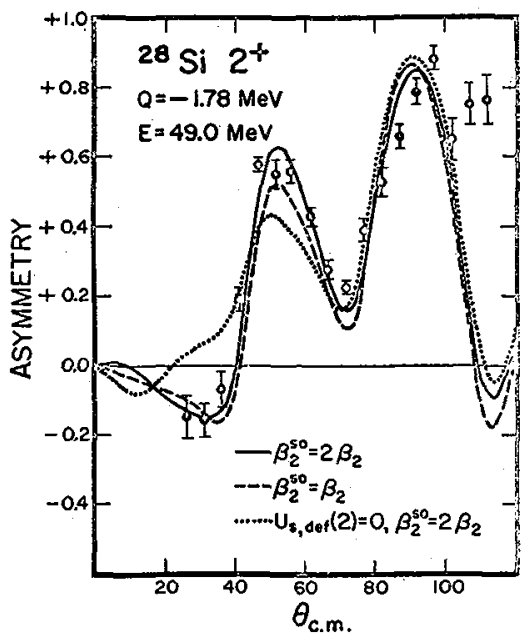

Fig. 2

Asymëtrie dans la diffusion $\mathrm{p}, \mathrm{p}^{+}$sur ${ }^{28} \mathrm{Si}$ à $49 \mathrm{MeV}$ ni veau $2+$ (Sheríf, réf. $[5 \mathrm{a}]$ ).

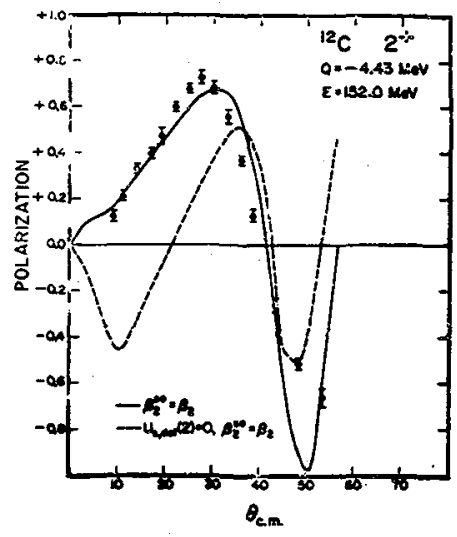

Fig. 3

Polarisation dans 1 a diffusion $p, p^{\prime}$ à $155 \mathrm{MeV}$ sur 1.2C niveau $2+$.

Cette forme de potentiel spin-orbite déformé rend bien compte des mesures d'asymétries faites par Lombard et a1. (communication) à $20 \mathrm{MeV}$ sur ${ }^{24} \mathrm{Mg},{ }^{26} \mathrm{Mg},{ }^{28} \mathrm{Si}$, mais, ici aussi, on doit donner à 


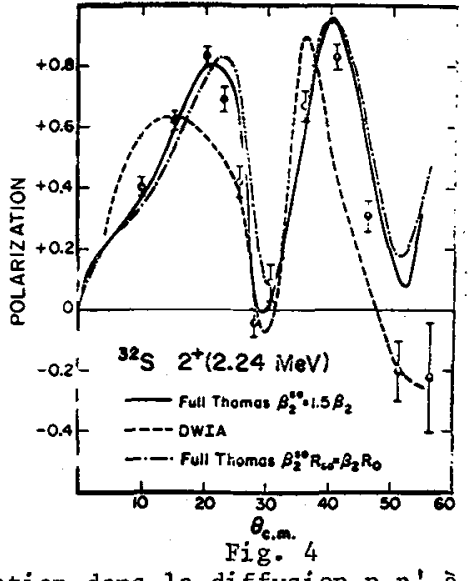

Polarisation dans la diffusion $\mathrm{p}, \mathrm{p}^{\prime}$ à $155 \mathrm{MeV}$ sur $32 \mathrm{~S}$ niveau $2+(\operatorname{réf.}[5 \mathrm{~b}]$ ).

$\mathrm{V}_{\mathrm{So}}$ une déformation plus grande. Raynal (communication) reprenant le traitement de 1 'interaction $\overrightarrow{\mathrm{L}} \overrightarrow{\mathrm{S}}$ nucléon nueléon dans une description microscopique, aboutit à la même forme que Shérif. Ceci entraîne, avec les approximations faites dans le calcul, une interaction spin orbite surtout importante dans l'excitation de configurations de protons, donc pour des noyaux à couche incomplète en protons, et qui doit dépendre de la nature des configurations des protons.

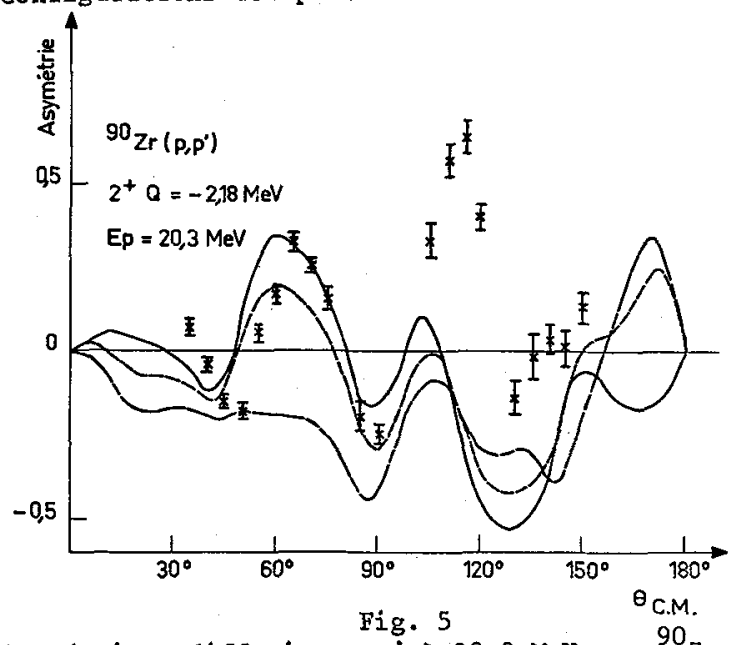

Asymétrie : diffusion p,p'à $20,3 \mathrm{MeV}$ sur ${ }^{90} \mathrm{Zr}$ niveau $2+$.

Pour compléter cette étude de la forme de 1'interaction à deux corps à utiliser en diffusion $\mathrm{p}, \mathrm{p}^{\prime}$ à moyenne énergie, il faut aussi préciser les termes qui entrainent un changement de spin isotopique; c'est dans ce but que Escudié et Tarrats (communication) ont étudié la transition du fondamenta1 $(1+\mathrm{T}=0)$ au premier état excité $(0+$ $T=1) \mathrm{de}^{14} \mathrm{~N}$.

Lorsque 1 'on aborde la diffusion inêlastique à plus haute énergie (supérieure à $100 \mathrm{MeV}$ ), on ne

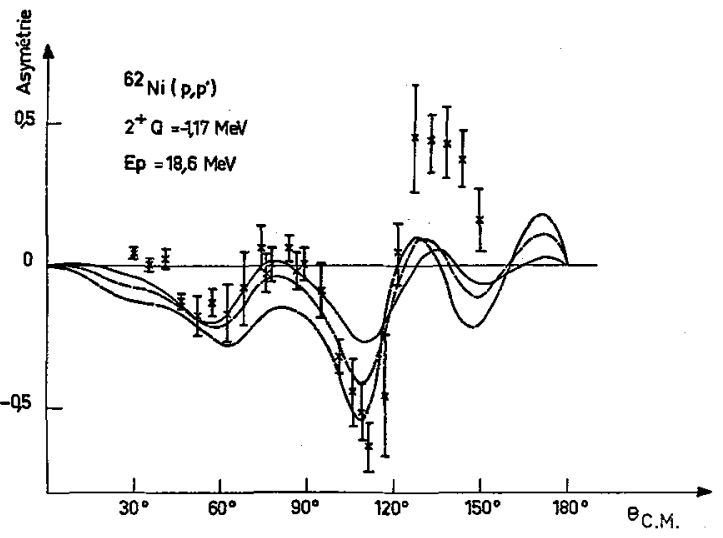

Fig. 6

Asymétrie : diffusion $p, p^{\prime}$ a $18,6 \mathrm{MeV}$ sur ${ }^{62} \mathrm{Ni}$ niveau 2+ (communication Raynal).

peut p1us prendre comne interaction à deux corps un potentiel qui rendrait compte uniquement de 1 'interaction dans 1 'état $S$; on utilise la matrice nucléon nuclêon libre (Hamada Johnston en général) dans une cinêmatique compensant en partie les effets hors couche d'énergie du choc du nucléon incident sur un nucléon lié au reste du noyau. Mais un traitement dans 1 'approximation d'impulsion en ondes planes ne suffit pas. Si les phënomènes de distorsion sont moins importants qu'à énergie plus basse, ils ne sont pas nêgligeables, et ont pour effet d'atténuer les sections efficaces d'un facteur qui crô̂t avec la masse du noyau ; à $150 \mathrm{MeV}$ : pax exemple de 1,5 environ pour les noyaux très 1êgers ${ }^{3} \mathrm{He},{ }^{3} \mathrm{H}$ (communication de Narboni et a1.), 2,2 pour ${ }^{12} \mathrm{C}, 3$ environ pour ${ }^{40} \mathrm{Ca}$.

Pour les diffusions aux grands angles, il faut tenix compte d'effets d'êchange mais le désaccord déjà apparu dans 1 'étude de la diffusion p,d [6]

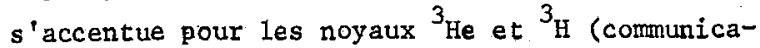
tion Narboni et a1.).

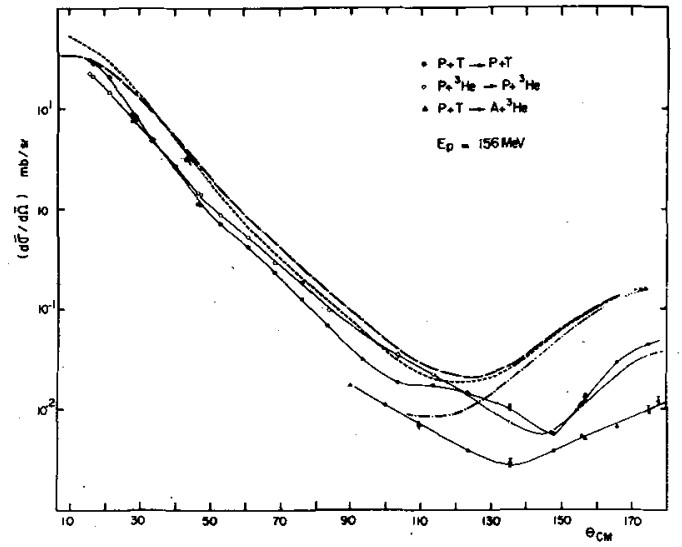

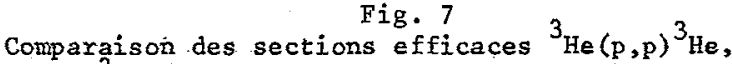
$\mathrm{T}(\mathrm{p}, \mathrm{n})^{3} \mathrm{He}$ (communication Narboni et al.). 
On peut essayer de faire un calcul des termes de diffusion multiple, come cela a été fait dans 1 'étude de la diffusion quasi élastique sur $D$,dont nous reparlerons tout à 1 'heure, en tenant compte uniquement de la diffusion élastique ou aussi de termes de diffusion inélastique lorsque le noyau présente des êtats excités très fortement couplés au fondamental [7]; mais un tel calcul demande de grosses approximations.

Pour les noyaux p1us lourds, la méthode habituellement utilisée, la D.W.I.A. a donné des résultats très satisfaisants pour ${ }^{40} \mathrm{Ca}$ [8] (a). Mais, pour de $p 1$ us grandes valeurs de $A$, la position des oscillations n'est pas bien rendue et surtout les sections efficaces décroissent trop rapidement pour les grands transferts d'impulsion; cet effet peut être dû̀ à l'approximation de pseudo por- têe nulle faite en.D.W.I.A. où l'impulsion transférée dans le choc nucléon nucléon est prise égale au transfert asymptotique. C'est cette approximation que Comparat (communication) a essayé de lever en tenant compte de la portée finie de l'interaction nucléon nucléon ; ce traitement encore incomplet améliore les résultats obtenus sux les $\mathrm{Sn}$.

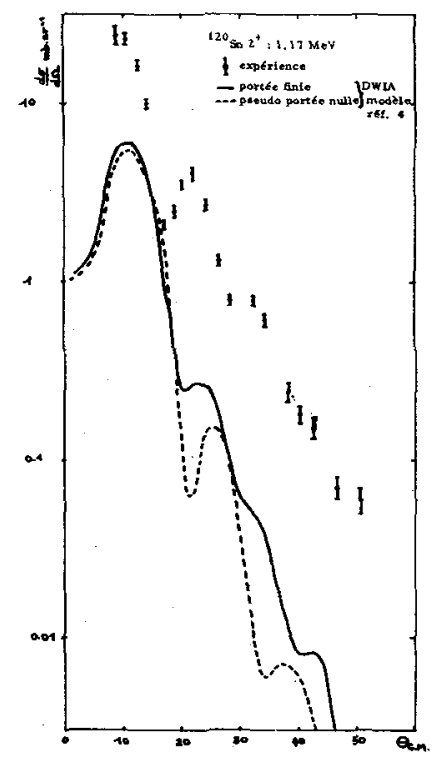

Fig. 8
Section efficace de diffusion p,p' sur ${ }^{120} \mathrm{Sn}$ niveau $2+$ (communication Comparat).

Le désaccord important qui subsiste dans ce cas entre rêsultats expērimentaux et thếoriques est surtout dû à 1 'insuffisance de la description microscopique utilisée pour les $\mathrm{Sn}$.
Nous avons traitê plus en détail la diffusion proton noyaux car la plupart des communications $s$ 'y rêféraient, mais il faut aussi aborder le problème de la diffusion $\alpha, \alpha^{\prime}$. Si dans ce cas l'interaction à deux corps $\alpha$ nuclểon peut être représentée de façon simple par un potentiel central avec partie rêlle et imaginaire, la forme la meilleure à donner à ce potentiel est encore mal définie et le problème du potentiel optique $\alpha$ noyau,à utiliser pour traiter les effets de distorsion, est imparfaitement résolu.

A ënergies moyennes, plusieurs familles de potentiels rendent également compte des résultats expérimentaux ; cela s'explique assez bien, la diffusion élastique $\alpha$ a lieu en surface, il suffit alors que ces potentiels aient même forme asymptotique pour qu'ils interprètent les expériences de diffusion élastique, mais ils peuvent diffêrer beaucoup à l'intérieur du noyau et n'être plus équivalents pour 1 'interprétation des diffusions inêlas tiques .

Des expériences de diffusion a faites par Brissaud et Tatischeff (communication) à $166 \mathrm{MeV}$, où 1a réaction est moins superficielle, semblent lever cette difficultê : un seul type de potentiel rend bien compte des résultats, on doit alors pouvoir par continuité choisir à plus basse énergie parmi les différents potentiels proposés; ces potentiels optiques ant pu être reconstruits à partis d'une interaction effective $\alpha$ nucléon proposée par d'autres auteurs [10] et les distributions de matière habituellement utilisées pour décrire les noyaux (modèle en couche, distribution de matière Hartree-Fock) ; la même interaction effective peut être utilisée dans la diffusion $\alpha, \alpha^{\prime}$. Si ces résultats se trouvent vérifiés par d'autres expêriences en cours, la diffusion $\alpha, \alpha^{\prime}$ pourra, elle-aussi, servir à tester les fonctions d'onde microscopiques des noyaux.

II. Diffusion quasi élastique.- Dans ce genre de réactions, la particule incidente proton, $\alpha$ (traitée come une particule) diffuse sur une particule du noyau, proton ou agrégat $(d, t, \alpha)$. La particule incidente et la particule choquée sont détectêes en coĩncidence et leur énergie est mesurée ; la réaction est alors complètement définie du point de vue cinematique. Si les effets du deuxième ordre sont négligeables, ou exactement calculables, on peut alors en dêduire la distribu- 
tion des impulsions de la particule choquée dans le noyau, et pour les particules agrégats, leur probabilité de présence à 1 'intérieur du noyau. Le mécanisme de telles réactions est-il actuellement suffisamment bien décrit pour que 1'on puisse atteindre ces résultats ?

Le meilleur test est $l^{\prime}$ étude de la réaction $D(p, 2 p) n$ pour laquelle la distribution des quantités de mouvement des nuclêons dans le deuton peut être considérée comme très bien connue (fonction d'onde de Hulthên pour le deuton) ; c'est pour cela que cette réaction a été, et est encore, étudiẻe dans un grand domaine d'énergie [1 $1 \mathrm{a}] \mathrm{de}-$ puis quelques $\mathrm{MeV}$ à, tout récenment, $1 \mathrm{GeV}$ [1 llb] et en particulier de 25 à $40 \mathrm{MeV}$ (communication de Durand et al.), à $155 \mathrm{MeV}$ (communication de Takéutchi et al.).

Dans tout ce domaine un calcul au premier ordre rend bien compte de la forme des distributions d'impulsion du nuclêon cible, mais pour les transferts d'impulsion importants, 1 'approximation du neutron spectateur ne suffit pas - il faut introduire 1 'interaction p.n. Par contre, au sommet de la courbe de distribution des moments, les sections efficaces calculées au premier ordre sont trop êlevées; le fait qu'entre 25 et $40 \mathrm{MeV}$ elless soient à peu près constantes se comprend : 1 a section efficace proton proton libre décroît avec 1 'énergie, mais aussi l'importance des termes de diffusion d'ordre supêrieur qui ont pour effet de diminuer cette section efficace; l'atténuation est très faible vers 150 ou $200 \mathrm{MeV}$ - elle crô̂t à nouveau aux grandes énergies pour atteindre un facteur d'environ 2 à $1 \mathrm{GeV}$. Les résultats expérimentaux obtenus à $155 \mathrm{MeV}$ par Takétutchi et al. (communication), comme ceux obtenus dans une autre cinématique par Morlet et a1. [12], montrent qu'un calcul tenant compte de tous les termes de diffusion double avec des approximations dépendant des énergies relatives des nucléons en interaction [13] rend compte presque complètement des rêsultats expërimentaux.

Les effets de distorsion sont évidemment bien plus importanţs pour les réactions $(p, 2 p)$ sur des noyaux plus lourds. Ce genre d'expériences a apportê, et apporte encore, des rểsultats extrêmement importants sur les énergies des couches occupées dans les noyaux [14] et (communication de Kullander et a1.). Par contre, lorsque l'on a voulu en déduire des renseignements sur les impulsions

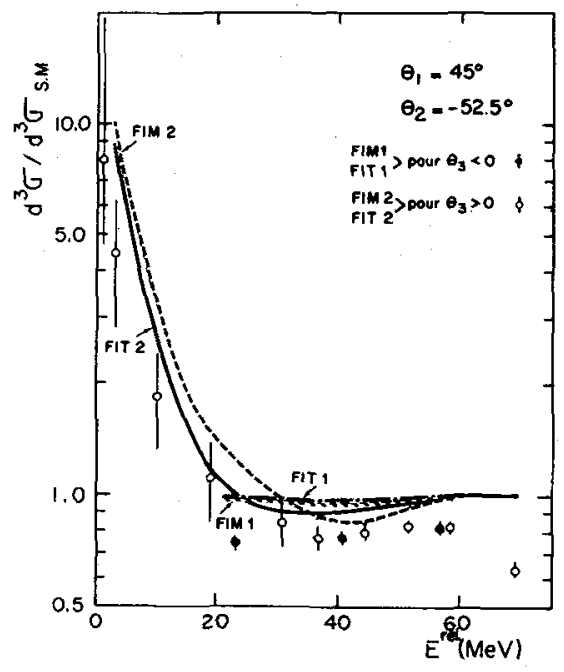

Fig. 9

Diffusion quasi êlastique $\mathrm{D}(\mathrm{p}, 2 \mathrm{p}) \mathfrak{n}$ à $155 \mathrm{MeV}$ (communication Takéutchi et a1).

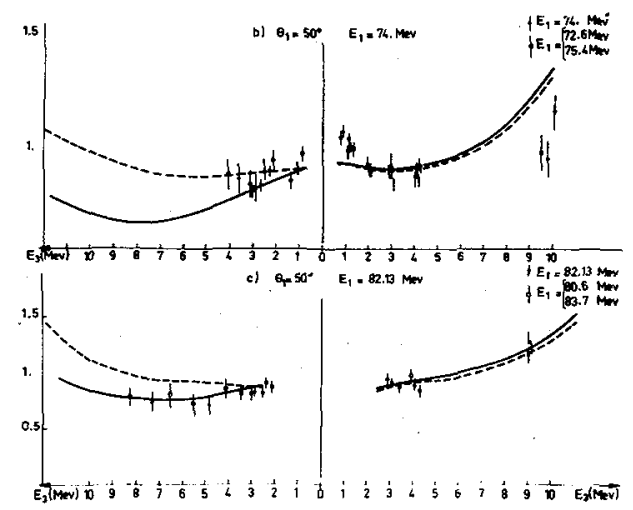

Fig. 10

Diffusion quasi êlastique $\mathrm{D}(\mathrm{p}, 2 \mathrm{p}) \mathrm{n}$ à $155 \mathrm{MeV}$ [12].

des nuclèons dans les couches $s, p, d$, on $s$ 'est heurté à la difficulté que les sections efficaces différentielles expérimentales (sauf pour la couche a) n'avaient pas la forme prévue par un calcul en ondes planes : courbes dissymétriques pour les nucléons $p$, minimum différent de 0 , déplacement de ce minimum. Ces effets très importants à $50 \mathrm{MeV}$ diminuent avec l'énergie de la réaction et sont faibles à $400 \mathrm{MeV}$ [15].

Un travail très complet de Hourani et al. (communication) et [16] sur ${ }^{12} \mathrm{C}$ et ${ }^{40} \mathrm{Ca}$ à $155 \mathrm{MeV}$ montre que,si on élimine les effets dus à une rêsolution angulaire insuffisante du dispositif expérimental, on obtient un bon accord pour la forme et la valeur absolue des sections efficaces, en géométrie coplanaire ou non, par un calcul en D.W.I.A. si les potentiels optiques distordants dans la voie 

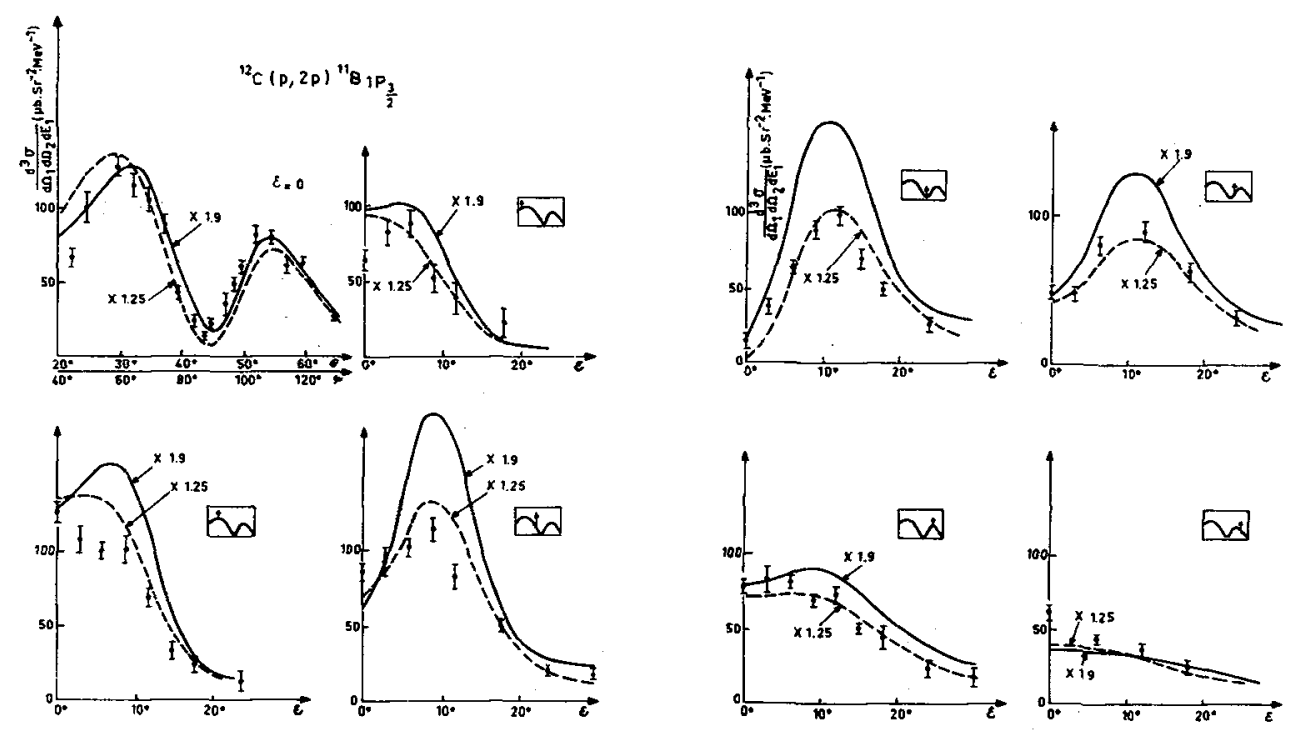

Fig. 11

Diffusion $(p, 2 p)$ sur ${ }^{12} \mathrm{C}$ (couche $1 p$ ) coplanaire et non coplanaire [16] (courbes calculées en ondes distordues, - - bon potentiel optique).

d'entrée et la voie de sortie sont des potentiels rendant bien compte des diffusions élastiques aux énergies correspondantes. Par contre, on ne retrouve pas, dans une géométrie non coplanaire, les forts effets d'interférence prévus par Jacob et Maris, si la réaction a lieu très en surface. Un traitement semblable peut être fait pour les réactions ( $p, p a)(p, p t)$ faites $155 \mathrm{MeV}$ sur des noyaux très lêgers ${ }^{6} \mathrm{Li},{ }^{7} \mathrm{Li}$ (communication de Bachelier et al.). La forme des distributions de quantitê de mouvement de 1 'agrégat par rapport au noyau cible correspond à ce que $1^{\prime}$ on peut prévoir ; on peut comparer les probabilitês de présence des différents agrégats mais il faut alors supposer que 1e reste du noyau est bien "spectateur" et que les effets du deuxième ordre sont faibles ou en tous cas identiques.

La réaction $(\alpha, 2 \alpha)$ sur les noyaux légers $a$, elle aussi, donnể lièu à un grand nombre de travaux, de 30 à $900 \mathrm{MeV}$ pour la particule incidente, et a étể traitée en prenant comme interaction $\alpha, \alpha$, 1'interaction $\alpha, \alpha$ libre phénoménologique; le travail fait à $55 \mathrm{MeV}$ par Gaillard et al. (communication) est interprétê dans cet esprit ; 1 'analyse est plus compliquée que dans $1 a$ diffusion $\left(p, 2_{P}\right)$ car les sections efficaces de diffusion $\alpha, \alpha$ libres prêsentent des résonances dans le domaine d'énergie relative considérée et on ne sait pas tenir compte des effets hors couche d'énergie. La distribution des quantités de mouvement des agrégats dépend de 1a méthode d'analyse; les probabilités de présen- ce de sous-structures $\alpha$ calculées dans différentes expériences de diffusion quasi élastique $(\alpha, 2 \alpha)$ ( $p, p \alpha$ ) dépend de l'énergie et de la nature de $1 \mathrm{a}$ particule incidente : cela montre, et ce n'est pas surprenant à de telles ênergies et pour des particules $\alpha$, que le premier terme de 1 'approximation d'impulsion ne suffit pas pour ce type d'expériences.

Jusqu'à présent nous nous sommes préoccupés de savoir coment traiter 1 'interaction nucléon nucléon pour atteindre 1 'interaction nucléon noyau ; nous allons maintenant nous poser la question suivante : 1 'étude de certaines interactions nucléon noyau peut-elle nous apporter des renseignements sur l'interaction nucléon nucléon; de façon plus précise, peut-on, à partir de l'interaction n.n.dans 1 'êtat final, avoir des renseignements sur l'interaction $n, n$ libre, difficile à mesurer directement. C'est dans ce but que Bachelier et al. (communication) ont étudié à 82 $\mathrm{MeV}$ en deutons les rëactions ${ }^{3} \mathrm{He}\left(\mathrm{d},{ }^{3} \mathrm{He}\right) \mathrm{pn}$, ${ }^{3} \mathrm{H}\left(\mathrm{d},{ }^{3} \mathrm{H}\right) \mathrm{pn},{ }^{3} \mathrm{H}\left(\mathrm{d},{ }^{3} \mathrm{He}\right) 2 \mathrm{n}$. Il est indispensable de faire des mesures comparatives, car si certaines réactions donnant lieu à une interaction dans I'état final telles les réactions type pick-up s'interprètent bien par la méthode habituellement utilisée de Watson et Migdal, les réactions avec échange $\left[\mathrm{d}\left({ }^{3} \mathrm{He}, t\right) \cdot 2 \mathrm{p}\right.$ par exemple] conduisent à des longueurs de diffusion $p, p$ beaucoup trop grandes [16]. Il faudra que les valeurs extraites des trois premières réactions citées soient en accord 
avec les valeurs bien connues des longueurs de diffusion $p, p$ et $p, n$ pour que $1^{\prime}$ on puisse faire confiance aux valeurs donnëes par la quatrième réaction pour $l^{\prime}$ interaction $n, n$.

Pour conclure sur une note optimiste, de grands progrès ont êté faits rêcemment dans le traitement de la diffusion inêlastique et aussi quasi êlastique ; dès maintenant des expériences de diffusion inélastique à moyenne énergie en protons, mais aussi en $\alpha$, peuvent servir à tester les descriptions microscopiques des noyaux.

\section{Bibliographie}

[1] SETH (K.), Nuc1. Phys., 1969, A138, 61 .

[2] COLE (R.K.) et al., Nucl. Phys., 1966, 75, 241.

[3] AMOS (K.A.) et al., Nucl. Phys., 1967, A94, 103.

[4] SCHAEFFER (R.), Thèse d'Etat, Orsay, 1969.

[5] (a) SHERIF (H.), Nucl. Phys., 1969, A131, 532 (b) SHERIF (H.) et BLAIR (J.S.), Nucl, Phys., $1970, \mathrm{~A} 140,33$.

[6] BENOIST-GUEUTAL (P.) et GOMEZ-GIMENO (F.), Phys. Letters, 1965, 13, 68 . KURODA (K.I.), thèse d'Etat, Orsay.

[7] TATISCHEFF (B.), Nuc1. Phys., 1967, A98, 384.

[8] haybron (R.M.) et Mc MANUS (H.), Phys. Rev., $1965,140,638$.

[9] WILlIS (A.) et a1., Nuc1. Phys., 1968, A112, 417.
[10] JACKSON (D.F.), Nuc1. Phys., 1969, A123, 273. BERNSTEIN (A.), Adv. in Nuc1. Phys., vo1. 3, 325.

[11] (a) KUCKES (A.F.) et al., Ann. of Phys., $1961,15,193$.

(b) SIMPSON (W.D.) et al., Nucl. Phys., 1970, A140, 201.

[12] MORLET (M.) et al., Nucl. Phys., 1969, A129, 177.

[13] L'HUililer (M.), Nuc1. Phys., 1969, A129, 196.

[14] RIOU (M.), Rev. Mod. Phys., 1965, 37, 375.

[15] JAMES (A.N.) et a1., Nuc1. Phys., 1969, A133, 89.

[16] HOURANI (E.), Thèse d'Etat, Orsay, 1970.

[17] MORTON (B.J.) et a1., Phys. Rev., 1968, 169, 825 . 